\title{
REPLY TO THE COMMENTS OF K. LEINFELDER ON "SURFACE ENERGY OF LIQUIDS ISOLATED IN NARROW CAPILLARIES"
}

\section{Received 10 August 1970}

Empirical evidence is available for the increase of liquid surface tension in isolated capillary spaces:

1) The increased strength of liquid bridges is a well known effect. The empirically verified Fisher equation was derived on this basis ${ }^{1}$ ). The high strength of thin bridges indicates high surface tensions.

2) Vapor pressure decreases with several liquids were demonstrated by Shereshefsky ${ }^{2}$ ). He measured vapor pressure lowerings, $\Delta P$, around $10-30$ times that calculated by using the Kelvin equation in the usual way. Table 1 was obtained using a modified Kelvin equation,

$$
R T \ln \left(P^{*} / P_{0}\right)=2 \gamma^{*} V / r
$$

for an isolated liquid. The liquid surface tension, $\gamma^{*}$, was obtained by integrating the equation

$$
\frac{\mathrm{d} \gamma}{\mathrm{d} A_{\mathrm{SL}}}=\frac{\left(F_{\mathrm{SV}}-F_{\mathrm{SL}}\right)}{A_{\mathrm{LV}}}
$$

for the case of a cylinder of 1 micron radius and liquid level $h$ as discussed in my paper $^{1}$ ).

The value calculated by using the Kelvin equation with a surface tension of 72 dyne $/ \mathrm{cm}$ is 0.017 , assuming a surface tension increase to $766 \mathrm{dyne} / \mathrm{cm}$, the $\Delta P$ value is increased around 10 times. Therefore the magnitude of the vapor pressure lowerings calculated is in reasonable agreement with Shereshefsky's data ${ }^{2}$ ).

TABLe 1

(Water at $18^{\circ} \mathrm{C}, P_{0}=15.477 \mathrm{~mm}$ )

\begin{tabular}{cccc}
\hline$h$ (microns) & $\gamma^{*}$ & $P^{*} / P_{0}$ & $\Delta P$ \\
\hline 0 & 72.0 & 0.9989 & 0.017 \\
1 & 130 & 0.9981 & 0.030 \\
2 & 198 & 0.9971 & 0.046 \\
5 & 411 & 0.9941 & 0.092 \\
10 & 766 & 0.9886 & 0.176 \\
\hline
\end{tabular}


The next comment is in regard to my assumption that the meniscus of a liquid bridge is cylindrical ${ }^{1}$ ). This was done in the paper to simplify the mathematics but recent experimental work done in our laboratory indicates the meniscus straightens out under tensile load as illustrated in fig. 1. At the maximum tensile force, which is what we are concerned with, the meniscus is quite straight. After this maximum force is exceeded the meniscus undergoes elongation and failure occurs.

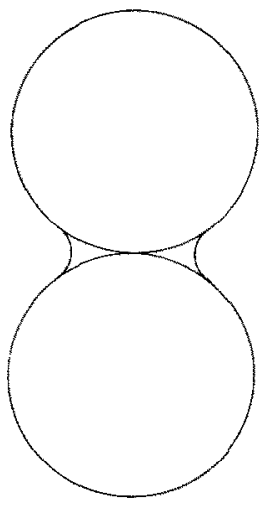

(a)

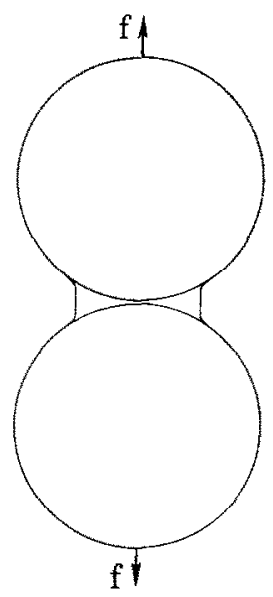

(b)

Fig. 1. Liquid meniscus of liquid bridge (a) straightens under applied force (b).

I will limit my reply to the last comment concerning anomalous water formation to effects an increase in surface tension might have on a condensate. During the formation of a phase, surface energy strongly affects the structure. In the case of a liquid, the fraction of holes of radius $r_{0}$ formed in a liquid of surface tension $\gamma^{*}$ is

$$
n / N=\exp \left[-4 \pi r_{0}^{2} \gamma / k T\right]
$$

at low pressures according to Frenkel ${ }^{3}$ ).

Fig. 2 is a plot of this fraction calculated as a function of $\gamma^{*}$. Assuming a hole radius of $1 \AA$, the fraction of holes in water at $20^{\circ} \mathrm{C}$ with a surface tension of $72 \mathrm{dyne} / \mathrm{cm}$ is calculated to be 0.11 or $11 \%$. Using a $\gamma^{*}$ value of $574 \mathrm{dyne} / \mathrm{cm}$, the same equation gives a hole fraction of $2 \times 10^{-8}$ or $0.000002 \%$. These calculations indicate that water formed under conditions of high surface tension would exhibit a negligible degree of porosity. Two paths are possible for the formation of such a condensate. Either the formation of a new dense phase with a density of $1.4 \mathrm{~g} / \mathrm{cm}^{3}$ for water cubically 


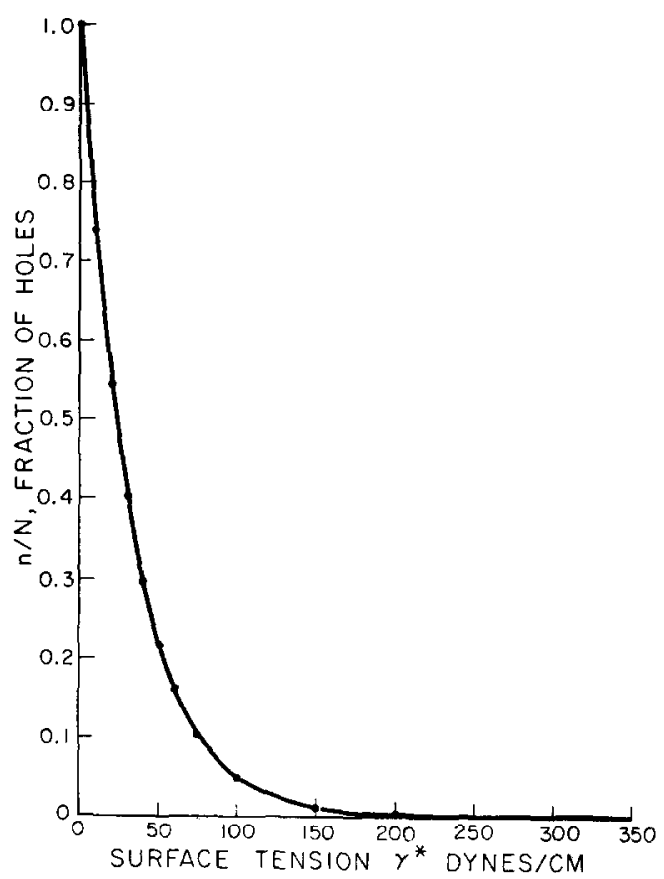

Fig. 2. Calculated fraction of holes formed in a liquid as a function of surface tension $\gamma^{*}$.
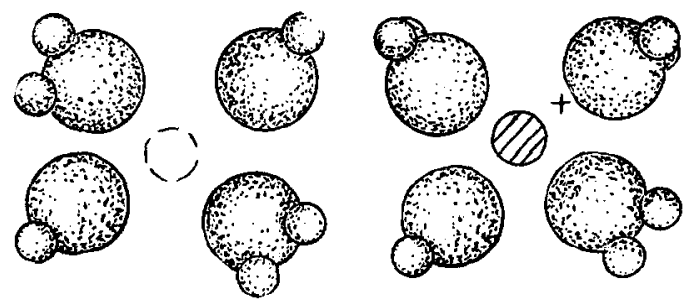

Fig. 3. Water molecules arranged around a hole (empty circle) and around an ion obtained from capillary wall.

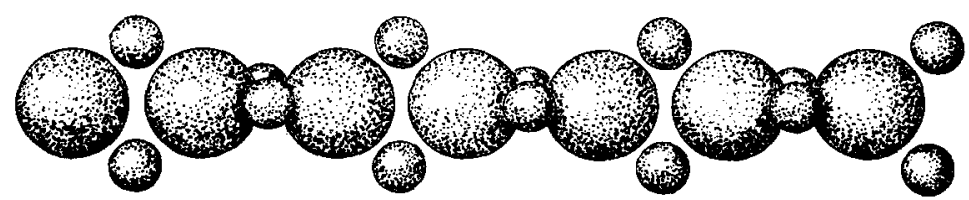

Fig. 4. A possible dense structure of anomalous water consisting of chains of dipoles. 
packed or the formation of a solution phase. As illustrated in fig. 3, a lower energy situation exists with solvent molecules arranged around an ion rather than a hole. If the dense pure phase forms, it is probably a polypole chain as illustrated in fig. 4. However if ions are present, there is little doubt that the solution phase will form since the energy required is considerably lower.

W. J. O'BRIEN

University of Michigan,

School of Dentistry,

Ann Arbor, Michigan 48104, U.S.A.

\section{References}

1) W. J. O'Brien, Surface Sci. 19 (1970) 387.

2) J. L. Shereshefsky, J. Am. Chem. Soc. 50 (1928) 2966.

3) J. Frenkel, Kinetic Theory of Liquids (Dover, New York, 1955) p. 180. 